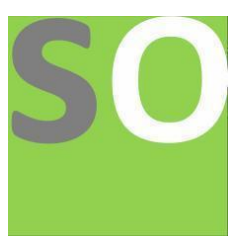

Article title: Norovirus: Vaccine Challenges and Development

Authors: Chloe Emehel[1], Akamu Ewunkem[2], Uchenna lloghalu[3]

Affiliations: Department of Biology, North Carolina A \& T State University, 1601 E Market Street, Greensboro NC USA[1], Department of Biological Science, Winston-Salem State University, 601 S Martin Luther King Jr Dr, Winston Salem NC USA[2]

Orcid ids: $0000-0002-9528-8240[1]$

Contact e-mail: chloeemehel@gmail.com

License information: This work has been published open access under Creative Commons Attribution License http://creativecommons.org/licenses/by/4.0/, which permits unrestricted use, distribution, and reproduction in any medium, provided the original work is properly cited. Conditions, terms of use and publishing policy can be found at https://www.scienceopen.com/.

Preprint statement: This article is a preprint and has not been peer-reviewed, under consideration and submitted to ScienceOpen Preprints for open peer review.

DOI: 10.14293/S2199-1006.1.SOR-.PPYGOKU.v1

Preprint first posted online: 18 November 2021

Keywords: norovirus, vaccine, challenges, development 


\section{Norovirus: Vaccine Development and Challenges}

Chloe Emehel ${ }^{1}$, Akamu Ewunkem², Uchenna lloghalu ${ }^{1 *}$

${ }^{1}$ Department of Biology, North Carolina A \& T State University, Greensboro NC USA,

${ }^{2}$ Department of Biological Science, Winston-Salem State University, Winston Salem NC

USA

Affiliation: North Carolina Agricultural and Technical State University, Greensboro NC 27411

Correspondence author's email: *ubiloghalu@ncat.edu 


\section{Abstract}

Noroviruses are a group of single-stranded RNA pathogens that cause acute gastroenteritis worldwide. Due to the high rates of mutation and evolution in the viruses, creating an effective vaccine to treat norovirus has been difficult. Factors such as the type of vaccine - live attenuated, subunit, or virus like particles - and target age group have limited progress in development. To better understand the best vaccine types and target groups for norovirus vaccine development, continuous research on the subjects will be encouraged. It will be shown that use of virus-like particles (VLPs) and a pediatric target group are the most effective options due to the versatility of VLPs and higher projected norovirus prevention rates for pediatric vaccination. The result of research will be useful to scientists creating norovirus vaccines and will help provide new perspectives for future research.

Key words: Norovirus, vaccine, challenges, development 


\section{Introduction}

Human Noroviruses (NoVs) are a group of non-enveloped RNA viruses (RNAVs) that are the leading causes of acute gastroenteritis and foodborne illnesses worldwide (Lloghalu, Khatiwada, Khatiwada, \& Williams, 2015). RNAVs are the culprits for the spread of pathogenic diseases due to their shorter generation times and high susceptibility to mutations and evolution. They are prone to mutations because of their lack of exonucleases that act as proofreaders during the replication process (CarrascoHernandez, Jácome, Vidal, \& Ponce de León, 2017). However, RNAVs benefit from mutations as the presence of more variants makes replication within the host more likely to occur. The other source of variability within RNAVs, evolution, is caused by recombination - the joining of RNA molecules from different genomes. Recombination allows for evolution by increasing the likelihood of favorable variants being produced and eradicating unfavorable traits as well (Carrasco-Hernandez, Jácome, Vidal, \& Ponce de León, 2017).

There are over 30 different Norovirus genotypes that affect humans and almost all have known recombinant variants (Parra, 2019). Based on the frequency at which these genotypes have recombined, some scientists question whether the prevalence of pointshift mutations (or genetic shift) has been underestimated (Ludwig-Begall, Mauroy, \& 
Thiry, 2018). This has been queried because of the speed at which NoVs can mutate, which is about $1.12-4.86^{\prime} 10^{-3}$ nucleotide substitutions/site/year (Tohma, et al., 2021). While both recombination and genetic shift produce unwanted variants of NoVs, this distinction could play a role in vaccine development and virus epidemiology. Lack of available therapies and the nature of the diseases caused by NoVs make vaccination an appealing strategy (Hallowell et al., 2019). This review analyzes the challenges scientists have faced regarding the development of an effective vaccine for NoVs. We also compile current knowledge in the fields of NoVs and vaccines to reveal gaps in what is known about the topics and discuss how to overcome these challenges and move towards next steps in production.

\section{Human Norovirus and its Epidemiology}

According to the Centers for Disease Control and Prevention (CDC), NoVs are responsible for about 19-21 million total illnesses, 2.3 million outpatient clinic visits, 109,000 hospitalizations, and 900 deaths per year in the United States alone (CDC, 2021). The low infectious dose of NoVs allows them to rapidly spread through the fecal and oral routes from direct contact with infected surfaces, food, feces, and airborne particles (lloghalu, Holmes, Khatiwada, \& Williams, 2019; Zhang, Fu, \& Hu, 2021). The most common symptoms are diarrhea, vomiting, abdominal pain, and nausea, which tend to occur 12-48 hours after exposure in symptomatic patients (Centers for Disease Control and Prevention, 2021; Robilotti, Deresinski, \& Pinsky, 2015). Similarly, these symptoms persist for a relatively short time of about two days on average. While many people make full recoveries from NoVs, pediatric and elderly patients have higher rates of death and more extreme symptoms due to developing or compromised immune systems, 
respectively. Elderly patients' compromised systems tend to worsen the symptoms of Norovirus and lengthen the duration of illness as well as a result from old age (Cardemil, Parashar, \& Hall, 2019). Generally, patients from less-developed countries also exhibit worse symptoms and higher death rates. This could be attributed to economic reasons such as contaminated food and water, restricted access to healthcare, and meager living conditions (Nguyen, Phan, Teng, Pu, \& Watanabe, 2017).

Presently, there is no treatment for norovirus; however, there are several vaccines in development (Riddle \& Walker, 2016). The most prevalent type of vaccine being developed utilizes virus-like particles (VLPs), although there are different proposed solutions that use P-particles and adenovirus (Esposito \& Principi, 2020). VLPs are the most proposed solution for vaccine development because they imitate the structure of the virus being used without involving the viral genome (Esposito \& Principi, 2020). Because VLPs lack the viral genome, they are not able to infect the host either making them safe and effective candidates for vaccine synthesis in addition to their ability to be produced in large quantities. (Qian, et al., 2020; Lucero, Vidal, \& O'Ryan G, 2018). P-particles, while not as commonly used, have also shown promising results towards NoVs vaccine development. P-particles are made by the domain of NoVs capsid proteins that interact with viral receptors. Additionally, NoVs P particles can be reproduced quickly in E. coli and are being considered for joint vaccine production combined with rotavirus, influenza, and hepatitis E (Esposito \& Principi, 2020; Riddle \& Walker, 2016; Wang, et al., 2014; Xia, et al., 2011). In the studies on influenza and hepatitis E dual vaccines, which used mice, results showed that each vaccine neutralized norovirus effectively. Furthermore, vaccines using recombinant adenovirus vectors have been developed. Adenovirus is an 
advantageous vector to use because of its abilities to promote immunity through pathogen-associated molecular patterns (PAMPS) - which are generally associated with activating immune responses (Chang, 2021). Between the three active components of the vaccines being developed - VLPS, P particles, and recombinant adenoviruses - all contain promising properties that could make an effective vaccine for NoVs with enough research and testing.

Given the spread and implications of NoVs in the United States and around the world, across all age groups, development of an effective vaccine has become vital for its control. To develop an effective vaccine, one must consider the recombination and mutation rates of NoVs, along with how fast the vaccine can be developed despite those factors (Parra, 2019; Tohma, et al., 2021). Recombination and mutations greatly affect essential components needed for a vaccine, such as the antigens needed to produce the most effective antibodies, researchers must work quickly in the areas of development, testing, and distribution before the virus significantly changes again. Other factors, such as the individual immune responses between age groups and most effective active components of the vaccine, also must be considered and have proven to be challenges in development (Zhang, Fu, \& Hu, 2021).

\section{Widely Used Vaccines}

Throughout the process of developing a Norovirus (NoV) vaccine, scientists have used various approaches in terms of the type of vaccine they attempt to develop. There have been three vaccine types that researchers focus on, which include VLPS, P-particles, or Adenovirus strains (Zhang, Fu, \& Hu, 2021). There are many other types that are not as 
widely considered but have been working to protect against other viruses. Types of vaccines range from those that use toxoids - which are pathogen protein toxins that have been inactivated - to mRNA and viral vectors. The most widely used and approved are live attenuated and subunit vaccines (Pollard \& Bijker, 2021).

\section{Live Attenuated Vaccines}

Live attenuated vaccines use weakened or inactivated forms of pathogenic germs to elicit an immune response in patients. These are considered very effective and have been used to prevent diseases like influenza, rotavirus, measles, and polio (Pollard \& Bijker, 2021). Although they are classified in the same group, many studies have separated live attenuated and inactivated vaccines into two separate categories. This is because there is much lower risk presented with inactivated vaccines; they only contain whole or components of killed pathogens (Pollard \& Bijker, 2021). Live attenuated vaccines, however, present many more risks, including reversion of the virus, higher risk to immunodeficient patients, mutation, and insufficient attenuation (Fuenmayor, Gòdia, \& Cervera, 2017; Noad \& Roy, 2003; Qian, et al., 2020).

Although live attenuated vaccines present more risks than inactivated ones, studies have shown a range of results from mild antibody responses and lower efficacy for inactivated vaccines to higher efficacy of inactivated vaccines compared to their live attenuated counterparts (Qian, et al., 2020). The most prevalent example of this can be seen in the evolution of the influenza vaccine. In a study by Belshe et al in 2007 , results showed higher efficacy of the live attenuated vaccine in experimental groups; specifically, they found that the group that received the live attenuated vaccine had almost $60 \%$ fewer influenza cases than the inactivated vaccine group (Belshe, et al., 2007). However, more recent studies by Chung et al and Jackson et al show opposite results. In both studies, 
$60 \%$ efficacy was observed with the inactivated influenza vaccine (Chung, et al., 2019; Jackson, et al., 2019). Based on this trend with the influenza vaccine, observations on the changes and improvements made with the influenza vaccine and vaccine technology over those 12 years should be made to create an effective norovirus vaccine, whether it is live attenuated or inactivated.

Studies have been increasing support for inactivated influenza vaccines over live attenuated ones. These are consistent with some animal and rotavirus-polio vaccine studies (Burke, Tate, Kirkwood, Steele, \& Parashar, 2019). Burke et al opined that various studies are mentioned where animals such as pigs, mice, rats, and rabbits, have been used. In all these studies, inactivated vaccines meant to protect against rotavirus and polio were used and produced various positive results. The inactivated rotavirus vaccine showed protective antibody reactions as well as comprehensive immunity. Further, the combined inactivated rotavirus-polio vaccine in question has shown promising results early on, with no obstruction of the immune response to either rotavirus or polio component (Burke, Tate, Kirkwood, Steele, \& Parashar, 2019). Given that many studies have shown more positive results in response to inactivated vaccines as opposed to live attenuated vaccines, inactivated vaccines may be recommended for a prospective norovirus vaccine.

\section{Subunit Vaccines and Virus-Like Particles}

Subunit vaccines have also become reputable in vaccine development and research. Subunit vaccines are those that use purified proteins, recombinant proteins, polysaccharides, or peptides to induce immunity. Influenza, hepatitis A and B, and typhoid, have been prevented using subunit vaccines, along with meningococcal and pneumococcal diseases (Pollard \& Bijker, 2021). Although subunit vaccines have been 
useful in modern medicine, they still have their weaknesses relative to live and inactivated vaccines. For example, most subunit vaccines require both higher doses to induce the same responses as live vaccines. Additionally, forms using proteins or polysaccharides are not always as effective as their live and inactivated counterparts (Pollard \& Bijker, 2021). Even though recombinant subunit vaccines do not allow for the development of virulent properties of viruses if their target proteins are misfolded, the immunogenicity of the vaccine is reduced (Azim, et al., 2019; Noad \& Roy, 2003). Polysaccharide vaccines - although providing substantial protection against some diseases - do not induce immune memory or sustained antibody production, which are both important for long term immunity. Further, polysaccharide vaccines have not shown immune response in infants under two years of age, which is the recommended timeframe for children to receive many vital vaccinations (Pollard \& Bijker, 2021).

Fortunately, the use of virus-like particles (VLPs) as subunits is becoming more frequently used for vaccines, especially those in development for NoVs. These VLPs allow for administration of smaller doses relative to many other subunit vaccines because of how VLPs can imitate the structures of their respective viruses; additionally, VLPs have been found to be safer than their live and inactivated counterparts due to their lack of genetic material (Noad \& Roy, 2003). VLPs are also able to be produced using many different methods such as with bacteria, yeast, insect and mammalian cells, and plants, with bacteria and yeast providing the most efficient forms of production. Developed vaccines that use VLPs include Hepatitis B, Malaria, Influenza A, and HIV (Fuenmayor, Gòdia, \& Cervera, 2017). Studies using VLPs for vaccination have also shown positive results for prevention of rotavirus and norovirus as well. For example, Groome et al. found 
that infants in South Africa were able to tolerate a rotavirus vaccine using VLPs at doses up to 60 micrograms (Groome, et al., 2017). Similarly, there is also a VP6 vaccine for rotavirus that uses norovirus VLPs to make a combination vaccine, which has shown positive immune responses in mice (Burke, Tate, Kirkwood, Steele, \& Parashar, 2019).

\section{Vaccines being considered for Noroviruses}

Currently, the are three main approaches being used for development of a NoVs vaccine: use of virus-like particles (VLPs), P-particles, and Adenovirus. VLPs are the most widely used and have made the most progress among different groups, with Takeda Pharmaceuticals and Vaxart Incorporated being two of the most recognized (Hallowell, Parashar, \& Hall, 2019). Takeda is developing a bivalent intramuscular vaccine and Vaxart is developing an oral monovalent vaccine; both of which are currently in clinical trials (Masuda, et al., 2018; Hallowell, Parashar, \& Hall, 2019). However, P-particles also have important qualities such as stability, ease of production, and preservation of norovirus surface antigens used to induce immunity (Xia, et al., 2011). Even with these two hopeful solutions, prior research has shown use of other types of subunits to be worth examining due to challenges of development and safety reasons. For safety, subunit vaccines may be better for norovirus vaccine development to prevent the symptoms that would most likely manifest after administration of a live vaccine.

\section{Challenges of Vaccine Development}

Regarding current challenges of development in addition to finding the right type of vaccine, researchers are mainly struggling to identify an appropriate target population. Inquiries have focused on the extremes of age: pediatric and elderly patients. Based on common knowledge surrounding vaccination already, infants and children are the 
targeted population for most vaccines that are provided today. Additionally, using infants and children as the target population of the norovirus vaccine may allow for limited transference to the general population (Cardemil, Parashar, \& Hall, 2019). Similarly, a model created by Steele et al. predicted vaccinating pediatric populations (children under 5 years) would prevent $33 \%$ and $60 \%$ of norovirus infections for lower vaccine efficacy and higher vaccine efficacy, respectively. However, the model for elderly vaccination (adults over 65 years) predicted prevention of only $17 \%$ and $38 \%$ of cases for respectively lower and higher vaccine efficacies (Steele, et al., 2016). The evidence suggesting pediatric vaccination as the best option continues when observing the incidence and hospitalization rates between children and elderly patients; the highest rates of disease and hospitalization are observed in young children, but the highest death rates are observed in elderly patients (Hallowell, Parashar, \& Hall, 2019). While this is not surprising, death rates from norovirus in elderly patients can eventually be lowered with pediatric administration of a norovirus vaccine, along with boosters as needed. If a conclusion on the ideal target population for norovirus vaccination cannot be reached soon, it may be wise to initially develop multiple norovirus vaccines for different age groups until more extensive research can be done.

Another challenge with development, or possibility, is the creation of a dual norovirus vaccine. A previously mentioned review by Burke et al. referred to a rotavirus vaccine in development that uses norovirus VLPS. This technology could lead to the creation of a dual vaccine for both rotavirus and norovirus, given that the norovirus VLPS used are effective at inducing immune responses for norovirus as well. Previous studies also investigated the possibility of developing dual vaccines for norovirus and influenza 
or hepatitis E (Xia, et al., 2011; Wang, et al., 2014). The advantage to developing a dual vaccine for influenza and norovirus would be the ability to incorporate it into pediatric vaccination schedules if that population is approved for a norovirus vaccine. Further, the study suggesting the dual vaccine investigates the use of P-particles, which is already known to be a more feasible option for norovirus vaccine development. On the other hand, there is not a hepatitis E vaccine currently approved for use in the United States, which will create some obstacles. Nevertheless, dual vaccines are an option that should be considered by researchers due to their ability to protect against multiple diseases, which has been seen in licensed vaccines that prevent diseases like tetanus, Diphtheria, and pertussis.

\section{Conclusion}

Noroviruses have high rates of recombination and reputations as the leading causes of gastroenteritis. Because of these factors, development of a vaccine has become increasingly important. The main vaccines being developed by both Takeda Pharmaceuticals and Vaxart Incorporated have used virus like particles, or VLPs. Use of VLPs has become the most prominent candidate for development of a norovirus vaccine due to their abilities to induce immune effects without introducing viral genetic material to patients at all. Although VLPs have made the most progress, it is still important to consider other types of vaccines that may use P-particles or adenoviruses, as these have been identified as likely candidates for norovirus vaccines as well. Additionally, dual vaccines have shown positive results, but there are very few studies despite this. Although Takeda and Vaxart have made considerable progress with developing a vaccine for noroviruses, 
it is still important to diversify the field and continue research on other types of vaccines that may be effective, from P-particles to dual vaccines.

A significant challenge of norovirus vaccine development for many scientists has been identifying a target age group. Based on previous vaccine studies, the target demographic should be children as opposed to adults or elderly patients. Children should be the target due to lower death rates due to the disease in their population, as well as better projected prevention rates compared to elderly patients. Norovirus vaccination of children would prove to be better in the long term, as their immunity would prevent the overall spread as they mature into adolescents and adults. Current research suggests that future research should continue development of VLP-based norovirus vaccines for children, but also continue work on alternative methods like dual vaccines as well. Dual vaccines should become more of a priority for efficiency reasons, especially since children tend to dislike shots in general. The development of a dual vaccine would allow the norovirus vaccine to be administered within the current vaccination schedule for children and save time and resources while doing so. 


\section{References}

Azim, K. F., Hasan, M., Hossain, M., Somana, S. R., Hoque, S. F., Bappy, M. I., . . Lasker, T. (2019). Immunoinformatics approaches for designing a novel multi epitope peptide vaccine against human norovirus (Norwalk virus). Infection, Genetics and Evolution, 112.

Belshe, R. B., Edwards, K. M., Vesikari, T., Black, S. V., Walker, R. E., Hultquist, M., ... Connor, E. M. (2007). Live Attenuated versus Inactivated Influenza Vaccine in Infants and Young Children. The New England Journal of Medicine, 685-696.

Burke, R. M., Tate, J. E., Kirkwood, C. D., Steele, A. D., \& Parashar, U. D. (2019). Current and new rotavirus vaccines. Current Opinion in Infectious Diseases, 435-444.

Cardemil, C. V., Parashar, U. D., \& Hall, A. J. (2019). Norovirus Infection in Older Adults Epidemiology, Risk Factors, and Opportunities for Prevention and Control. Infectious Disease Clinics of North America, 839-870.

Carrasco-Hernandez, R., Jácome, R., Vidal, Y. L., \& Ponce de León, S. (2017). Are RNA Viruses Candidate Agents for the Next Global Pandemic? A Review. ILAR Journal, 343-358.

Centers for Disease Control and Prevention. (2021, March 5). Norovirus. Retrieved from Centers for Disease Control and Prevention: https://www.cdc.gov/norovirus/index.html

Chang, J. (2021). Adenovirus Vectors: Excellent Tools for Vaccine Development. Immune Network, 1-11.

Chung, J. R., Flannery, B., Ambrose, C. S., Bégué, R. E., Caspard, H., DeMarcus, L., . . Fry, A. M. (2019). Live Attenuated and Inactivated Influenza Vaccine Effectiveness. Pediatrics, 1-9.

Cortes-Penfield, N. W., Ramani, S., Estes, M. K., \& Atmar, R. L. (2017). Prospects and Challenges in the Development of a Norovirus Vaccine. Clinical Therapeutics, 1537-1549.

Esposito, S., \& Principi, N. (2020). Norovirus Vaccine: Priorities for Future Research and Development. Frontiers in Immunology, 1-9.

Fuenmayor, J., Gòdia, F., \& Cervera, L. (2017). Production of virus-like particles for vaccines. New Biotechnology, 174-180.

Groome, M. J., Koen, A., Fix, A., Page, N., Jose, L., Madhi, S. A., . . Cryz, S. (2017). Safety and immunogenicity of a parenteral P2-VP8-P[8] subunit rotavirus vaccine in toddlers and infants in South Africa: a randomised, double-blind, placebo-controlled trial. The Lancet Infectious Diseases, 843-853.

Hallowell, B. D., Parashar, U. D., \& Hall, A. J. (2019). Epidemiologic challenges in norovirus vaccine development. Human Vaccines and Immunotherapeutics, 1279-1283.

Iloghalu, U., Holmes, B., Khatiwada, J., \& Williams, L. L. (2019). Selected Plant Extracts Show Antiviral Effects against Murine Norovirus Surrogate. Advances in Microbiology, 372384.

Jackson, M. L., Chung, J. R., Jackson, L. A., Phillips, C. H., Benoit, J., Monto, A. S., . . Fry, A. (2019). Influenza Vaccine Effectiveness in the United States during the 2016-2017 Season. Clinical Infectious Diseases, 1798-1806.

Lloghalu, U., Khatiwada, P., Khatiwada, J., \& Williams, L. (2015). Phytochemicals: Natural Remedies for Emerging Viral Infection. Medicinal and Aromatic Plants, 1-7.

Lucero, Y., Vidal, R., \& O'Ryan G, M. (2018). Norovirus vaccines under development. Vaccine, 5435-5441. 
Ludwig-Begall, L. F., Mauroy, A., \& Thiry, E. (2018). Norovirus recombinants: recurrent in the field, recalcitrant in the lab - a scoping review of recombination and recombinant types of noroviruses. Journal of General Virology, 970-988.

Masuda, T., Lefevre, I., Mendelman, P. M., Sherwood, J., Bizjajeva, S., \& Borkowski, A. (2018). Immunogenicity of Takeda's Bivalent Virus-like particle (VLP) Norovirus Vaccine (NoV) candidate in Children from 6 months up to 4 years of Age. Zurich: Takeda Pharmaceuticals AG.

Micoli, F., Bagnoli, F., Rappuoli, R., \& Serruto, D. (2021). The role of vaccines in combatting antimicrobial resistance. Nature Reviews Microbiology, 287-302.

Nguyen, G. T., Phan, K., Teng, I., Pu, J., \& Watanabe, T. (2017). A systematic review and metaanalysis of the prevalence of norovirus in cases of gastroenteritis in developing countries. Medicine, 1-6.

Noad, R., \& Roy, P. (2003). Virus-like particles as immunogens. Trends in Microbiology, 438-444.

Parra, G. I. (2019). Emergence of norovirus strains: A tale oftwo genes. Virus Evolution, 1-9.

Pollard, A. J., \& Bijker, E. M. (2021). A guide to vaccinology: from basic principles to new developments. Nature Reviews Immunology, 83-100.

Prymula, R., Povey, M., Brzostek, J., Cabrnochova, H., Chlibek, R., Czajka, H., . . Wys. (2021). Ten-year follow-up on efficacy, immunogenicity and safety of two doses of a combined measles-mumps-rubella-varicella vaccine or one dose of monovalent varicella vaccine: Results from five East European countries. Vaccine, 2643-2651.

Qian, C., Liu, X., Xu, Q., Wang, Z., Chen, J., Li, T., . . Xia, N. (2020). Recent Progress on the Versatility of Virus-Like Particles. Vaccines, 1-14.

Riddle, M. S., \& Walker, R. I. (2016). Status of vaccine research and development for norovirus. Vaccine, 2895-2899.

Robilotti, E., Deresinski, S., \& Pinsky, B. A. (2015). Norovirus. Clinical Microbiology Reviews, 134164.

Steele, M. K., Remais, J. V., Gambhir, M., Glasser, J. W., Handel, A., Parashar, U. D., \& Lopman, B. A. (2016). Targeting pediatric versus elderly populations for norovirus vaccines: a model-based analysis of mass vaccination options. Epidemics, 42-29.

Tohma, K., Lepore, C. J., Martinez, M., Degiuseppe, J. I., Khamrin, P., Saito, M., . . Manee. (2021). Genome-wide analyses of human noroviruses provide insights on evolutionary dynamics and evidence of coexisting viral populations evolving under recombination constraints. PLoS Pathogens, 1-35.

Wang, L., Cao, D., Wei, C., Meng, X.-J., Jiang, X., \& Tan, M. (2014). A dual vaccine candidate against norovirus and hepatitis $E$ virus. Vaccine, 445-452.

Xia, M., Tan, M., Wei, C., Zhong, W., Wang, L., McNeal, M., \& Jiang, X. (2011). A candidate dual vaccine against influenza and noroviruses. Vaccine, 7670-7677.

Zhang, M., Fu, M., \& Hu, Q. (2021). Advances in Human Norovirus Vaccine Research. Vaccines, 1-11. 